\title{
Delayed complications of intravaginal slingplasty multifilament tape: two case reports
}

\section{Sivakumar S. Balakrishnan \\ Department of Obstetrics and \\ Gynecology, Penang Medical College; \\ Department of Obstetrics and \\ Gynecology, Hospital Pulau Pinang, \\ Penang; Penang Adventist Hospital \\ Penang, Malaysia}

\section{Abstract}

We would like to present two cases of delayed complications of intravaginal slingplasty (IVS) suburethral sling in Penang, Malaysia. There were two patients who were referred to us for problems subsequent to suburethral sling surgery using the IVS sling. These patients were reviewed and subsequently treated. The first patient presented with a bleeding granuloma. She underwent surgery and intra operatively the granuloma was excised. On exploration there was remnant of the tape bilaterally. The tape was easily excised with traction. A diagnostic cystoscopy did not show any abnormality. The second patient presented with a severe sling exposure. This patient also had surgery to excise the sling. It was easily dislodged with traction and removed completely. There were pus collections on one side. On subsequent review both patients were well with no more vaginal bleeding or discharge. Both patients however remained continent. This report is to show IVS sling complications may present in different manner and the need for attending physician to be aware of these presentations. They should be aware of the delayed presentation of these complications.

\section{Introduction}

Intravaginal slingplasty (IVS) sling is multifilament polypropylene tape that was first reported in 1996 as a treatment of urinary stress incontinence. It was found to be equally effective as TVT ${ }^{\mathrm{TM}}$ in treating urinary stress incontinence. ${ }^{1,2}$ Longer term follow up of this sling surgery however noted evidence of higher risk of mesh exposure and other complications. ${ }^{3,4}$ This is most likely due to the multifilament nature of the tape which has smaller pore size ( $<75$ microns) that prevents macrophages from infiltrating through the sling. This promotes infection and subse- quently non incorporation of the sling into the tissues. ${ }^{3,4}$ This eventually leads to an increased risk of vaginal exposure of the sling. The usual presentation is of mesh exposure. However we would like to present two cases of mesh complications presenting in different manner. One was in the form of severe exposure and the other in the form of a granuloma.

\section{Case Reports}

\section{Case \#1}

The first patient presented to us in 2005 for complaints of stress urinary incontinence. She was a 52-year old lady with underlying diabetes mellitus controlled with oral hypoglycemics. She underwent a midurethral sling procedure using the retropubic approach with the IVS sling.

This patient developed early exposure after 6 months after surgery. The exposed part of mesh was trimmed in the clinic. She was subsequently well for two years. Then she presented herself to the clinic with intermittent vaginal bleeding. She had attained menopause at the age of 54 years. Vaginal examination showed that she had developed a vaginal granuloma at the level of midurethral placement of the sling (Figure 1).

She was initially treated with vaginal estrogen cream by the attending gynecologist but the granuloma was persistent with continued intermittent bleeding. She was then subsequently referred to the author, who had managed cases of IVS sling exposure previously. Examination showed the granuloma was present but there was no obvious mesh exposure. The complication was classified as 1B/T4/S1 using the International Urogynecological Association (IUGA) classification. ${ }^{5}$ The patient was counseled for exploration and excision of the tape. She was counseled that removal of the tape may result in recurrent urinary stress incontinence. The patient underwent the surgery in early February 2008. Intra-operatively the granuloma was excised with a circumferential incision around the granuloma. On exploration under the excised granuloma, there was remnant of the tape bilaterally seen (Figure 2). The tape was easily dislodged on traction using an Ellis forceps and was removed in pieces. A diagnostic cystoscopy did not show any abnormality. The incision was closed with interrupted sutures using absorbable suture. Post operatively she was put on broad-spectrum prophylactic antibiotics for seven days. She recovered well and the vaginal wound healed completely. The histology of the excised tissues confirms fibrogranulation tissue with foreign body reaction. She did not require any further estrogen local application .She has regular follow up till now and she has been well and remains continent.
Correspondence: Sivakumar S. Balakrishnan, Department of Obstetrics and Gynecology, Penang Medical College, 4, Jalan Sepoy Lines, 10450, Penang, Malaysia.

Tel. +60.42263459 - Fax: +60.42284285 .

Mobile: 60124299003.

E-mail: kum67kris@yahoo.com; sivakumar@pmc.edu.my

Key words: intravaginal slingplasty, exposure, presentation.

Received for publication: 5 August 2013.

Revision received: 19 November 2013.

Accepted for publication: 20 November 2013.

This work is licensed under a Creative Commons Attribution NonCommercial 3.0 License (CC BYNC 3.0).

CC Copyright S.S. Balakrishna., 2013

Licensee PAGEPress, Italy

Urogynaecologia 2013; 27:e7

doi:10.4081/uij.2013.e67

\section{Case \#2}

The second patient presented to another institution because of prolapse and stress urinary incontinence. She was a 39 -year old lady with no underlying comorbidities, who underwent a Manchester repair and midurethtral sling with the IVS sling using the retropubic approach. This patient presented after 1 year with obvious severe mesh exposure (Figure 3 ). The tape was exposed for about $3 \mathrm{~cm}$ in length. She had also complained of persistent vaginal discharge. After consulting a few other physicians, this patient was then referred to the author for further management. The mesh complication was classified as 3B/T4/S1 using the IUGA classification. ${ }^{5}$ She was counseled for excision of the tape. She was also advised that the removal might make her incontinent again.

This patient had surgery in March 2012. Upon exploration, the sling was clearly visible. The sling was easily dislodged with traction on the exposed tape using an Ellis forceps. The tape was completely removed (Figure 4 ). There was a sterile pus collection on the left side of the removed tape. The vaginal exposure area was excised and edges closed with interrupted sutures using absorbable suture. The patient was also given broad-spectrum antibiotic for seven days. She did not require any further treatment. This patient recovered well and the vaginal wound healed completely. Currently she is still on follow up. She also remains continent. 


\section{Discussion}

Here we have two patients with mesh complications with multifilament sling presenting in two different ways. One was in the form of granuloma and the other the more common presentation of mesh exposure. We are now well aware of the risks of mesh exposure particularly using the multifilament mesh.,3,4,7

The most common form of exposure reported is the direct exposure of the mesh as illustrated by the second case. However we need to be aware that the mesh complications can present in a variety of ways. It can also present in the form of retropubic abscess and ischiorectal abscess. ${ }^{4,8}$ The delayed presentations of these problems are also significant to note. It can also present with frequent vaginal discharge, urinary tract infections. It may present in unusual way such as vaginal bleeding due to granuloma formation as shown in the first case. Therefore it is

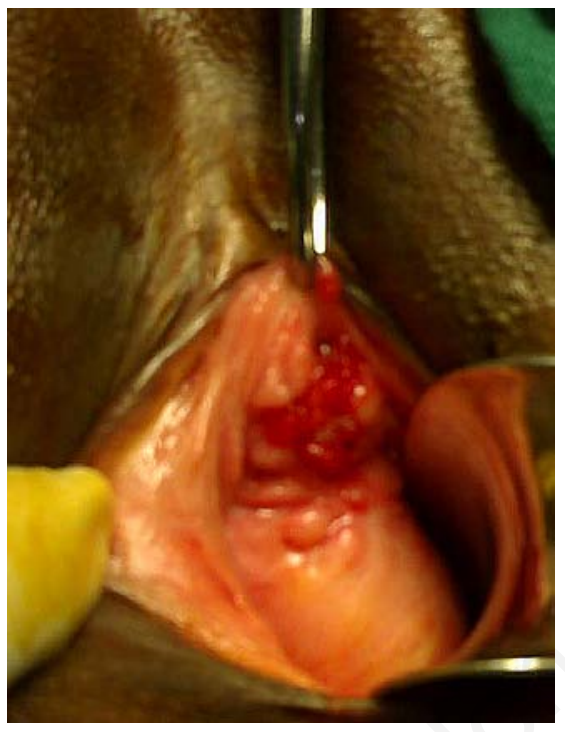

Figure 1. Granuloma.

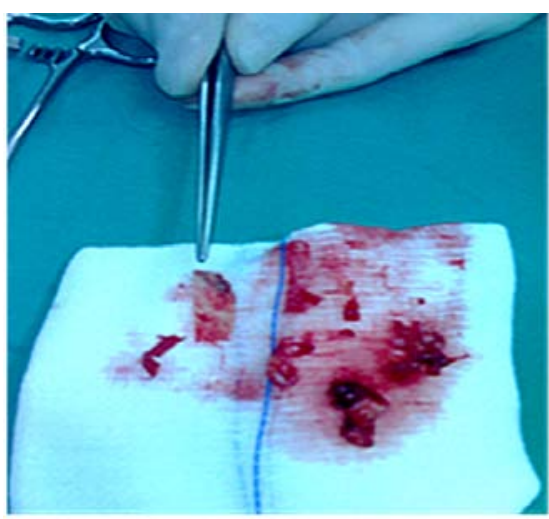

Figure 2. The piecemeal remnants of the intravaginal slingplasty sling. prudent for physicians who are seeing patients presenting with lower urinary tract symptoms or other pelvic symptoms such as pain, abnormal vaginal bleeding and vaginal discharge after a midurethral sling surgery to evaluate them thoroughly. History should be elicited in regards to type of sling used in order to identify the type used. This will help in detecting complications related with the multifilament tapes. Even though the use of the multifilament sling have been largely abandoned, awareness regarding the delayed complications will significantly attending physicians to help patients by avoiding delays to refer to a physician with experience dealing with this problem. An interesting fact is that both patients remained continent even after complete excision of the slings. The patients would have been expected to have recurrence of urinary stress incontinence. The histological findings of fibrogranulation tissue may suggest that fibrosis has occurred around the midurethra at the sling placement site even though the sling

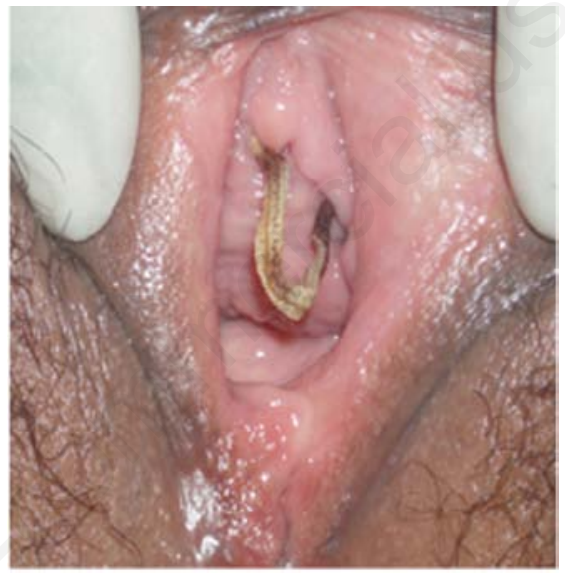

Figure 3. The intravaginal slingplasty sling exposure.

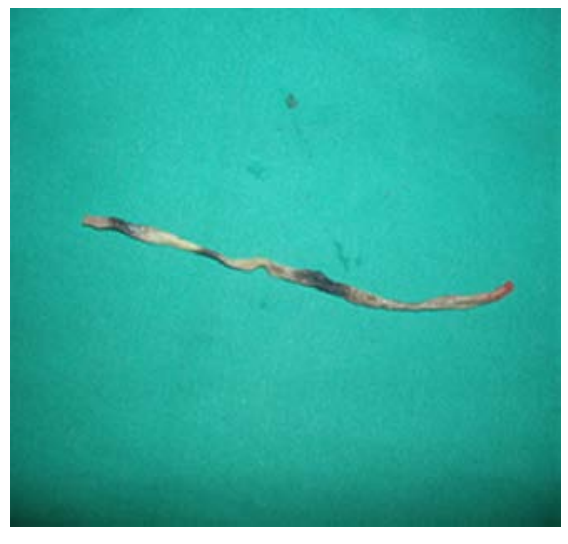

Figure 4. The complete removal of the sling. was not incorporated into the tissues. We could only speculate that the fibrous tissues are providing the support during the increase in the intra abdominal pressure similar to a sling. This may have rendered them continent.

\section{Conclusions}

The two reported cases remind us to look for potential delayed complications of the IVS. Even though the use of IVS has been discontinued, newer physicians need to be aware of these complications and be vigilant with patients presenting with complications after a midurethral sling procedure. We would also encourage appropriate training for surgeons using slings especially in recognizing mesh exposure and handling its related complications.

\section{References}

1. Petros PP. The intravaginal slingplasty operation, a minimally invasive technique for cure of urinary incontinence in the female. Aust N Z J Obstet Gynaecol 1996;36:453-61.

2. Lim YN, Muller R, Corstiaans A, et al. Suburethral slingplasty evaluation study in North Queensland, Australia: the SUSPEND trial. Aust N Z J Obstet Gynaecol 2005;45:52-9.

3. Kim JC, Chung BS, Choi JB, et al. A safety and quality of life analysis of intravaginal slingplasty in female stress incontinence: a prospective, open label, multicenter, and observational study. Int Urogynecol J Pelvic Floor Dysfunct 2007;18:1331-5.

4. Balakrishnan S, Lim YN, Barry C, et al. Sling distress: a sub analysis of the IVS tapes from the SUSPEND trial. Aust N Z J Obstet Gynaecol 2007:47:496-8 .

5. Haylen BT, Freeman RM, Swift SE, et al. An International Urogynecological Association (IUGA)/International Continence Society (ICS) joint terminology and classification of the complications related directly to the insertion of prostheses (meshes, implants, tapes) and grafts in female pelvic floor surgery. Int Urogynecol J 2011;22:3-15.

6. Lo TS, Ashok K. Recurrent mesh erosion and retropubic abscess following anterior intravaginal slingplasty. J Obstet Gynaecol Res 2011;37:363-5.

7. Nyyssönen V, Talvensaari-Mattila A, Santala M. Intravaginalslingplasty sling is associated with increased risk of vaginal erosion. Acta ObstetGynecol Scand 2009;88:1222-6.

8. Jha S, Radley S, Shorthouse A. Infected midurethral tape presenting as an ischiorectal abscess. Int Urogynecol J Pelvic Floor Dysfunct 2008;19:877-9. 